\title{
Effect of dexmedetomidine on the development
} \section{of mechanical allodynia and central sensitization in chronic post-ischemia pain rats}

This article was published in the following Dove Press journal: Journal of Pain Research

\section{Jinseok Yeo'}

Sungsik Park ${ }^{2}$

'Department of Anesthesiology and Pain Medicine, Kyungpook National University Chilgok Hospital, School of Medicine, Kyungpook National University, Daegu, Korea; ${ }^{2}$ Department of Anesthesiology and Pain Medicine, Kyungpook National University Hospital, School of Medicine, Kyungpook National University, Daegu, Korea
Correspondence: Jinseok Yeo

Department of Anesthesiology and Pain Medicine, Kyungpook National University Chilgok Hospital, School of Medicine, Kyungpook National University, 807 Hoguk-ro, Buk-gu, Daegu 4I404, Korea

Tel +82532002644

Fax +82532002027

Email jsyeo@knu.ac.kr
Purpose: Complex regional pain syndrome type 1 (CRPS I) is an intractable neuropathic pain syndrome. Chronic post-ischemia pain (CPIP) model is an animal model of CRPS I which is produced by ischemia-reperfusion (IR) injury of the hind limb. Dexmedetomidine (DEX) is a selective and potent $\alpha 2$ adrenergic receptor agonist with analgesic and protective effects following an IR injury. We hypothesized that DEX protects the development of mechanical allodynia and central sensitization in CRPS I. Therefore, we evaluated the dose-related protective effect of DEX in a CPIP model.

Methods: We divided 45 rats into five groups: sham, CPIP, CPIP + DEX $10 \mu \mathrm{g} / \mathrm{kg}, \mathrm{CPIP}+$ DEX $50 \mu \mathrm{g} / \mathrm{kg}$, and CPIP + DEX $100 \mu \mathrm{g} / \mathrm{kg}$. Rats in the sham group underwent sham surgery, and the other rats received CPIP injury. One hour before reperfusion or end of sham surgery, normal saline was injected into the rats in the sham and CPIP groups, and DEX (designated dose) was injected into the rats in the other groups. All rats were evaluated for the withdrawal threshold of both hind paws before surgery and 1,3, and 7 days after surgery. Phosphorylation of $N$-methyl-D-aspartate receptor subunits (pGluN1) and phosphorylation of extracellular signalregulated kinases (pERK) in the spinal cord were measured 3 days after surgery.

Results: Administration of DEX before reperfusion showed a significant increase in the withdrawal threshold in both hind paws and a significant decrease of the expressions of pGluN1 and pERK in CPIP rats dose dependently $(P<0.05)$.

Conclusion: DEX may inhibit the development of mechanical allodynia and central sensitization in CPIP rats.

Keywords: allodynia, central sensitization, chronic post-ischemia pain, dexmedetomidine

\section{Introduction}

Complex regional pain syndrome (CRPS) is a chronic pain condition characterized by spontaneous pain, hyperalgesia, allodynia, and abnormal vasomotor and sudomotor activities. ${ }^{1}$ Complex regional pain syndrome type I (CRPS I) usually occurs after fracture, crushing injury, or soft tissue injury without clinically verified nerve damage. The pathophysiological mechanisms ofCRPS I are multifactorial, such as peripheral and central sensitization, inflammation, altered sympathetic function, and altered somatosensory representation in the brain. ${ }^{2}$

Chronic post-ischemia pain (CPIP) model has been considered an animal model of CRPS I. ${ }^{3}$ Ischemia-reperfusion (IR) injury of a hind limb effectuates the CPIP model and shows persistent mechanical and cold allodynia without nerve injury. Central sensitization is an amplification of neural signaling by neural plasticity, and an essential mechanism in persistent pain. ${ }^{4}$ It reduces pain thresholds and increases responses to noxious stimuli. Phosphorylation of extracellular signal-regulated kinases (pERK) is 
a marker of neural plasticity which acts to mediate central sensitization and phosphorylation of $N$-methyl-D-aspartate (NMDA) receptors in the spinal cord is indicative of central sensitization. $^{5}$

Dexmedetomidine (DEX) is a selective and potent $\alpha 2$ adrenergic receptor agonist. It is highly lipid-soluble and readily crosses the blood-brain barrier following systemic administration. DEX penetrates the central nervous system after systemic administration and demonstrates a sedative effect. ${ }^{6}$ It has an analgesic effect related to its $\alpha 2$ adrenergic receptor binding properties in inflammatory and neuropathic pain ${ }^{7,8}$ and demonstrated an organ-protective effect against IR injury in the brain, heart, kidney, and skeletal muscle in animal models. ${ }^{9-12}$

We hypothesized that preventive administration of DEX before reperfusion could modulate pain and central sensitization in CPIP rats via its analgesic and organ-protective effects. Thus, we evaluated the effect of DEX on the development of mechanical allodynia and central sensitization in CPIP rats via changes in hind limb withdrawal thresholds, as well as the expression of phosphorylated NMDA receptor subunit 1 (pGluN1) and pERK in the spinal cord.

\section{Methods}

\section{Animals and experimental protocol}

The study protocol was approved by the Kyungpook National University Institutional Animal Care and Use Committee (Figure 1). A total of 45 male Sprague Dawley rats (320-340 g) were used (Koatech, Gyeonggi-do, Korea) in this study.
The rats were acclimatized to acryl cages for 7 days before surgery. All rats were housed at the animal facility under specific pathogen-free conditions and received care according to the National Institutes of Health guidelines on laboratory welfare. Rats were randomly assigned to one of five groups ( $\mathrm{n}=9$ ): 1) sham, 2) CPIP, 3) CPIP + DEX $10 \mu \mathrm{g} / \mathrm{kg}$, 4) CPIP + DEX $50 \mu \mathrm{g} / \mathrm{kg}$, and 5) CPIP + DEX $100 \mu \mathrm{g} / \mathrm{kg}$. Rats in the sham group underwent sham surgery, and rats in the other groups underwent the CPIP procedure. All rats were treated 1 hour before reperfusion or at the end of surgery. Rats in the vehicle group received normal saline $\left(0.1 \mathrm{~cm}^{3}\right)$ intraperitoneally. Rats in the CPIP + DEX $10 \mu \mathrm{g} / \mathrm{kg}, \mathrm{CPIP}+$ DEX $50 \mu \mathrm{g} / \mathrm{kg}$, and CPIP + DEX $100 \mu \mathrm{g} / \mathrm{kg}$ groups received DEX (Predex ${ }^{\circledR}$, DEX HCl; Hospira, Lake Forest, IL, USA) at the designated doses intraperitoneally. We chose the doses of DEX according to a previous study. ${ }^{12}$

\section{Animal model}

CPIP rats as described by Coderre et $\mathrm{al}^{3}$ were used in this study. We anesthetized rats over a 3-hour period using an intraperitoneal injection of sodium pentobarbital (Entobar; Hanlim Pharm, Seoul, Korea). We used pentobarbital $50 \mathrm{mg} /$ $\mathrm{kg}$ to induce anesthesia and $20 \mathrm{mg} / \mathrm{kg} / \mathrm{h}$ to maintain anesthesia. After that, a Nitrile 70 Durometer O-ring (O-Rings West, Lynnwood, WA, USA) with a 5.5-mm internal diameter was placed around the left hind limb just proximal to the ankle joint and left for 3 hours. We removed the O-ring at the end of 3 hours, and normal blood flow to the injured limb was

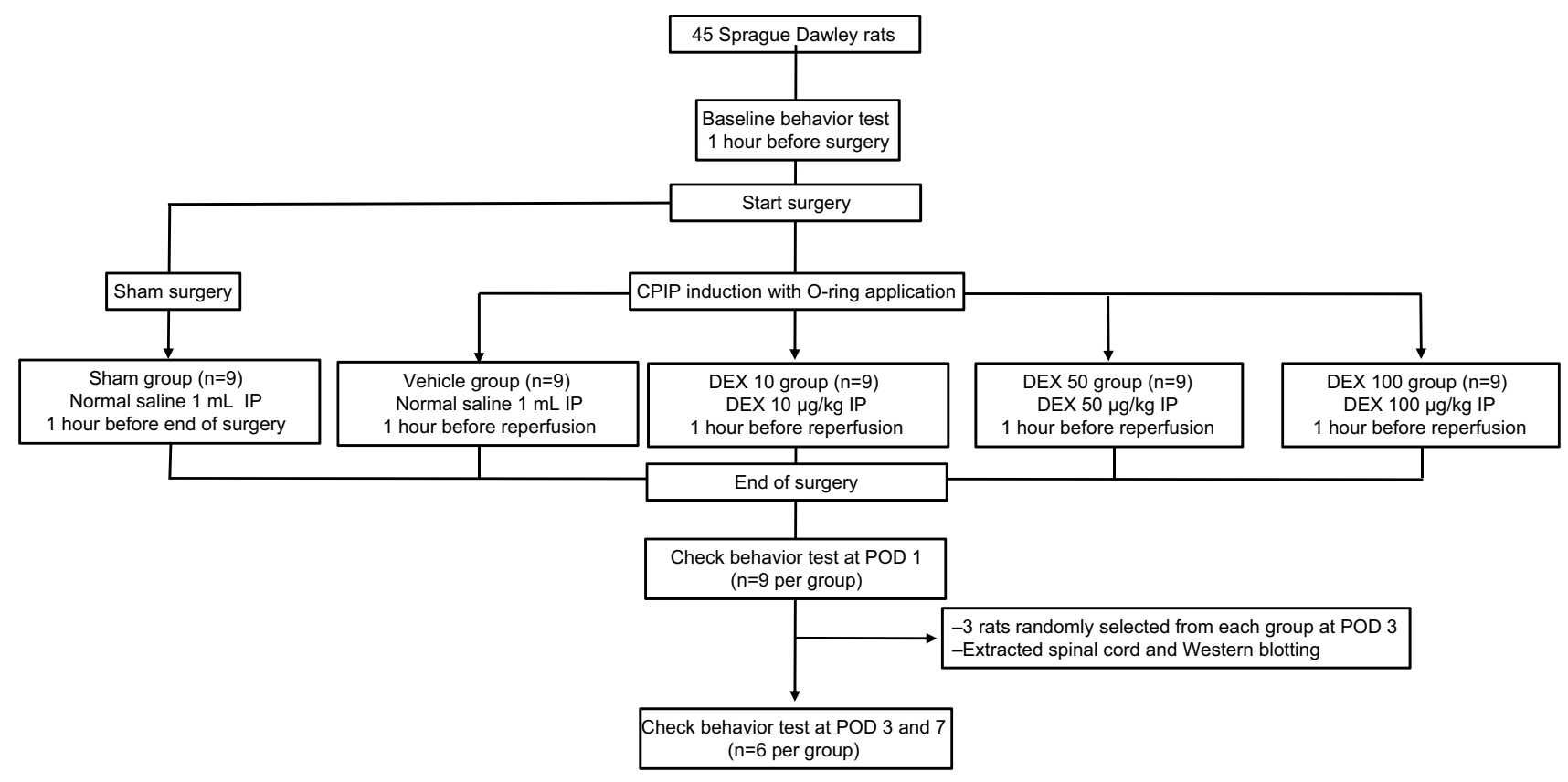

Figure I Study flow.

Abbreviations: CPIP, chronic post-ischemia pain; DEX, dexmedetomidine; IP, intraperitoneal injection; POD, postoperative day. 
reestablished. Rats in the sham group underwent the anesthesia procedure, but a cut O-ring was placed loosely around the ankle for 3 hours.

\section{Assessment of mechanical allodynia of hind paws}

Mechanical allodynia was assessed 1 hour before surgery (baseline), and 1, 3, and 7 days after surgery. A researcher blinded to the treatments conducted all behavioral tests. Rats were placed in a transparent acrylic cage $\left(23 \times 16 \times 24 \mathrm{~cm}^{3}\right)$ with a steel mesh floor and allowed to acclimatize to the environment for 1 hour per day for 2 days before the experiment, and 30 minutes before the behavior test on the test day. Withdrawal thresholds to mechanical stimulation were measured according to the $50 \%$ withdrawal response to Von Frey filaments with logarithmic incremental stiffness (Stoelting, Wood Dale, IL, USA) using the up-down method. ${ }^{13}$ Von Frey filaments were pushed vertically to the center of the plantar surface of the hind limb until the filament bent. Withdrawal movement, flinching, stamping, or licking of the hind limb within 10 seconds of the filament application were considered positive responses. We started with a filament producing a force of $2 \mathrm{~g}$; lower-intensity filaments were applied after each positive response and higher-intensity filaments were applied after each negative response. The lowest and highest stimulus intensities were 0.25 and $15 \mathrm{~g}$. If a negative response occurred at the highest stimulus intensity, or a positive response occurred at the lowest stimulus intensity, the value was taken as the cutoff value. Withdrawal responses were measured at 5-minute intervals, alternating between the right and left paws.

\section{Western blot analysis of pGluNI and PERK}

We randomly selected three rats from each group before surgery. The selected rats did not undergo the behavioral pain test. Three days after surgery, the rats were anesthetized with an intraperitoneal injection of sodium pentobarbital and rapidly sacrificed. The L4-6 section of the spinal cord was rapidly extracted after laminectomy, and the left side of the spinal cord was frozen in liquid nitrogen. The samples were dissolved in a tissue lysate buffer containing a protease inhibitor cocktail tablet (Hoffman-La Roche Ltd., Basel, Switzerland). Proteins were quantified from supernatants using Bradford dye assays (Bio-Rad Laboratories Inc., Hercules, CA, USA). Protein samples were heated at $100^{\circ} \mathrm{C}$ for 5 minutes and loaded onto a 10\% SDS-polyacrylamide gel before being transferred onto a nitrocellulose membrane.
The membrane was blocked in 3\% nonfat milk with Trisbuffered saline ( $50 \mathrm{mM}$ Tris $\mathrm{pH} 7.4$ and $10 \mathrm{mM} \mathrm{NaCl}$ ) at room temperature for 1 hour and the blots were incubated with an anti-phospho-NR1 antibody (1:1,000, Ser897, ABN99; EMD Millipore, Billerica, MA, USA), anti-pERK antibody (1:1,000, SC-7383; Santa Cruz Biotechnology Inc., Dallas, TX, USA), and anti $\beta$-actin antibody $(1: 5,000, \beta$-actin, SC-47778; Santa Cruz Biotechnology Inc.) at $4^{\circ} \mathrm{C}$ overnight. After washing with Tris-buffered saline $(50 \mathrm{mM}$ Tris $\mathrm{pH} 7.4$ and $10 \mathrm{mM} \mathrm{NaCl}$ ), the membrane was incubated with horseradish peroxidase-conjugated secondary anti-mouse antibody (1:10,000, SC-2031; Santa Cruz Biotechnology Inc.) for 1 hour at room temperature and developed in enhanced chemiluminescence solution for 1 minute. The blots were exposed to autoradiographic film. The densities of the protein blots were quantified using LabWorks 4.5 software (Ultra-Violet Products Ltd, Cambridge, UK).

\section{Statistical analysis}

Data are presented as the mean \pm standard error of the mean and were analyzed using SPSS Statistics for Windows, version 21.0 (IBM Corporation, Armonk, NY, USA). Data were verified for normality using Shapiro-Wilk tests. Statistical analyses for differences in changes over time in multiple groups were performed using two-way ANOVA with one repeated factor (time), followed by Tukey's test for post hoc analyses. Between-group differences were tested using one-way ANOVA (when comparing more than two groups) followed by Tukey's test for post hoc analyses. For all tests, a two-tailed $P<0.05$ was considered indicative of statistically significant difference.

\section{Results}

\section{Hind paw mechanical allodynia}

We evaluated the neuropathic behavior of CPIP rats and investigated whether DEX can modulate neuropathic behavior by measuring mechanical withdrawal thresholds. The mechanical withdrawal thresholds for both hind paws at baseline did not differ between the groups. Rats in the sham group did not show significant changes in the mechanical withdrawal thresholds in both hind paws over the 7-day study period. For rats in the CPIP + DEX $50 \mu \mathrm{g} / \mathrm{kg}$ and CPIP + DEX $100 \mu \mathrm{g} /$ $\mathrm{kg}$ groups, the withdrawal thresholds after reperfusion of the ipsilateral hind limbs were significantly decreased compared to those in the sham group over the 7-day study period, but the withdrawal thresholds of the contralateral hind limbs showed no significant changes compared to those in the sham group. Rats in the CPIP and CPIP + DEX $10 \mu \mathrm{g} / \mathrm{kg}$ groups 
showed significant decreases of the withdrawal thresholds of both hind paws over the 7-day study period when compared to baseline values. Rats in the CPIP + DEX $50 \mu \mathrm{g} / \mathrm{kg}$ and CPIP + DEX $100 \mu \mathrm{g} / \mathrm{kg}$ groups showed significantly higher withdrawal thresholds in both hind paws over the 7-day study period when compared to the vehicle group (Figure 2).

\section{Measurement of pGluNI and pERK}

To evaluate the extent of central sensitization and the alleviating effect of DEX on central sensitization in CPIP rats, we analyzed pGluN1 and pERK in the spinal cord. Rats in the vehicle groups showed significant increases of pGluN1 and pERK expression in the ipsilateral spinal cord when compared to rats in the sham group. Rats in the CPIP + DEX 50 $\mu \mathrm{g} / \mathrm{kg}$ and CPIP + DEX $100 \mu \mathrm{g} / \mathrm{kg}$ groups showed significant reductions of pGluN1 and pERK expression in the ipsilateral spinal cord compared to the vehicle group (Figure 3).

\section{Discussion}

We successfully reproduced the CPIP model by inducing mechanical allodynia in both hind paws and demonstrated that administration of DEX before reperfusion in CPIP rats
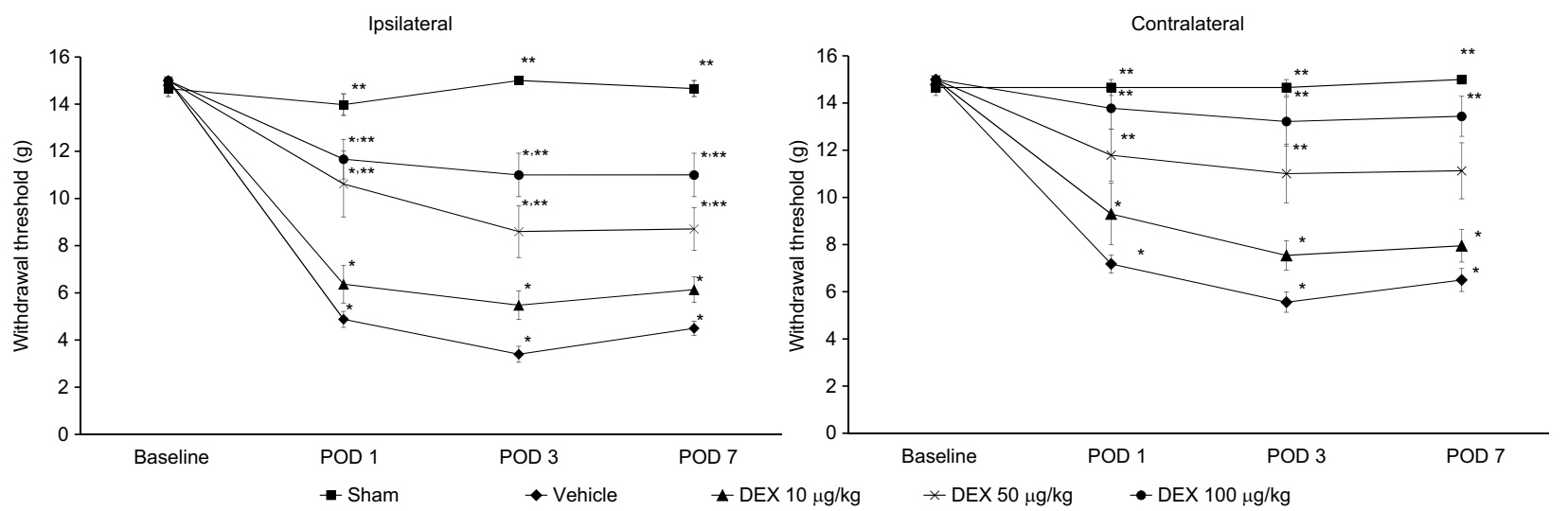

Figure 2 Mechanical allodynia of ipsilateral (left) and contralateral (right) hind limbs assessed using a Von Frey filament.

Notes: Compared with the sham group, the vehicle, DEX $10 \mu \mathrm{g} / \mathrm{kg}$, DEX $50 \mu \mathrm{g} / \mathrm{kg}$, and DEX $100 \mu \mathrm{g} / \mathrm{kg}$ groups showed significantly decreased withdrawal thresholds for 7 days after surgery. In each group, testing began with nine animals per group; three were killed for Western blot at POD 3 and the remaining six continued with behavioral testing until POD 7. The sham group was treated with saline (I mL, IP) I hour before the end of anesthesia. The vehicle group was treated with saline (I mL, IP) I hour before reperfusion. The DEX $10 \mu \mathrm{g} / \mathrm{kg}$, DEX $50 \mu \mathrm{g} / \mathrm{kg}$, and DEX $100 \mu \mathrm{g} / \mathrm{kg}$ groups were treated with 10,50 , and $100 \mu \mathrm{g} / \mathrm{kg}$ of DEX I hour before reperfusion, respectively. Withdrawal thresholds were assessed I hour before anesthesia (baseline), and POD I, POD 3, and POD 7 after surgery. Data are expressed as mean \pm standard error of the mean. *Indicates significant difference from the sham group. $* *$ Indicates significant difference from the vehicle group. $P<0.05$ was considered statistically significant. Abbreviations: DEX, dexmedetomidine; IP, intraperitoneal injection; POD, postoperative day.
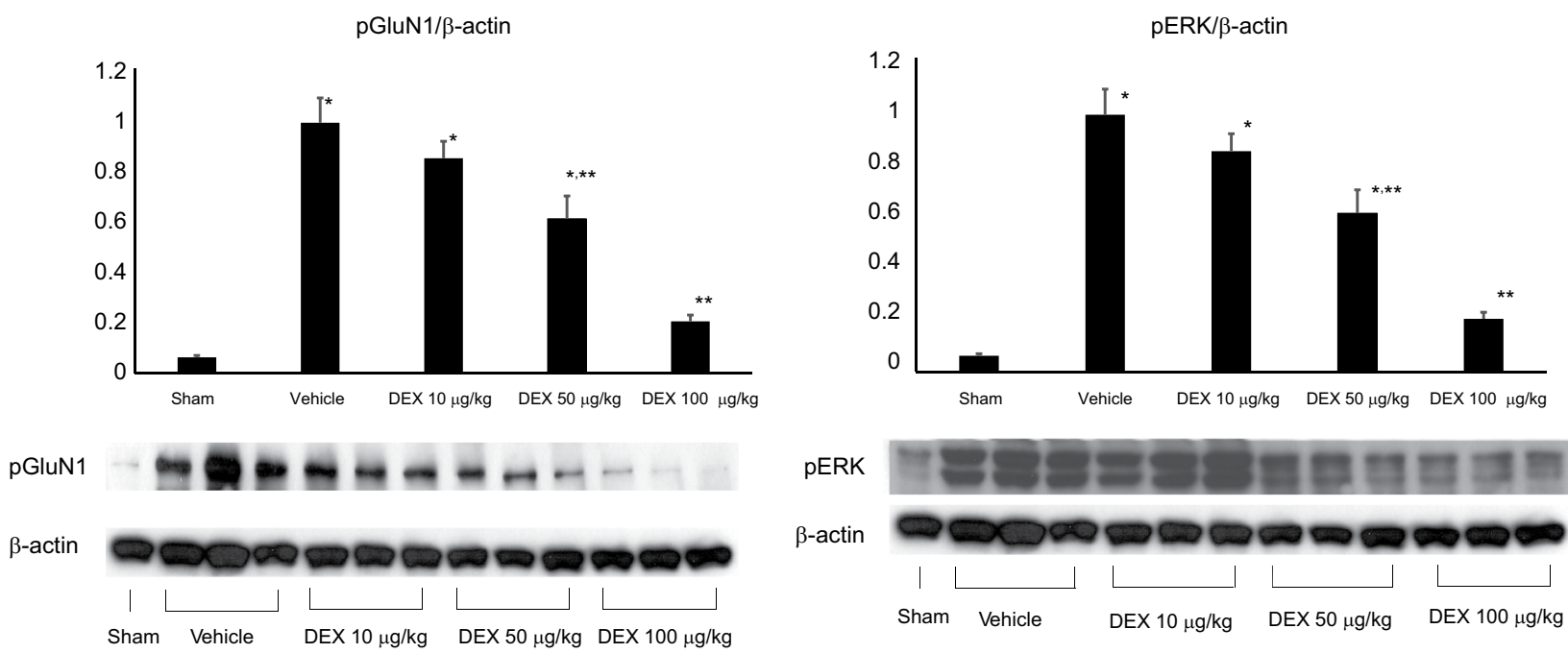

Figure 3 Changes in the relative density of pGluNI and pERK protein in the ipsilateral spinal cord (L4/6) measured by Western blotting at POD 3.

Notes: Three rats were randomly selected from each group. The sham group was treated with saline (I mL, IP) I hour before the end of anesthesia. The vehicle group was treated with saline $(I \mathrm{~mL}$, IP) I hour before reperfusion. The DEX $10 \mu \mathrm{g} / \mathrm{kg}$, DEX $50 \mu \mathrm{g} / \mathrm{kg}$, and DEX $100 \mu \mathrm{g} / \mathrm{kg}$ groups were treated with I0, 50 , and I00 $\mu \mathrm{g} / \mathrm{kg}$ of DEX I hour before reperfusion, respectively. Data are expressed as mean \pm standard error of the mean. *Indicates significant difference from the sham group. **Indicates a significant difference from the vehicle group. $P<0.05$ was considered statistically significant.

Abbreviations: DEX, dexmedetomidine; pGluNI, phosphorylation of $N$-methyl-D-aspartate receptor subunit I; pERK, phosphorylation of extracellular signal-regulated kinases; IP, intraperitoneal injection; POD, postoperative day. 
attenuated the development of mechanical hyperalgesia and central sensitization by inhibition of the extracellular signal-regulated kinase (ERK) signaling pathway and NMDA receptors.

Allodynia in CPIP rats develops from the day of reperfusion and peaks on the third day after reperfusion. It persists for 4 weeks. Activation of pGluN1 in CPIP rats also peaks on the third day after reperfusion. ${ }^{13}$ In our study, mechanical allodynia and activation of pGluN1 and pERK reduced in the group that was administered $>50 \mu \mathrm{g} /$ $\mathrm{kg}$ of DEX. However, we did not find any significant differences between $50 \mu \mathrm{g} / \mathrm{kg}$ and $100 \mu \mathrm{g} / \mathrm{kg}$ of DEX. This result suggests that $50 \mu \mathrm{g} / \mathrm{kg}$ of DEX is enough to prevent mechanical allodynia and central sensitization in CPIP rats. Contralateral allodynia in CPIP rats is evidence of central sensitization and less severe than ipsilateral allodynia. ${ }^{14}$ In our study, we observed less severity of contralateral allodynia in all the CPIP rats and no differences in mechanical allodynia between the sham group and $>50 \mu \mathrm{g} / \mathrm{kg}$ of DEXinjected CPIP groups.

Prolonged ischemia and reintroduction of oxygenated blood into the ischemic limb causes IR injury. IR injury is a complex phenomenon including intracellular injury processes and inflammatory responses after prolonged ischemia. ${ }^{15}$ Ischemia causes energy depletion, cell damage, accumulation of toxic metabolites, elevation of tissue acidity, and activation of phospholipase and lysozymes. ${ }^{16}$ Reperfusion worsens the injury by inducing cellular infiltration and generating ROS. ${ }^{17}$ IR injury produces local inflammatory processes, which increases proinflammatory cytokines, such as tumor necrosis factor- $\alpha$ (TNF- $\alpha$ ) and IL- $1 \beta$ and causes apoptosis, endothelial cell dysfunction, and necrosis. ${ }^{18}$ Persistent inflammation after IR injury may activate nociceptors in the muscle, periosteum, and skin. Inflammatory stimuli can activate the ERK signaling pathway in the spinal cord, and the activated ERK signaling pathway contributes to persistent hypersensitivity. ${ }^{19}$ Activation of nociceptors of the ischemic limb may also lead to central sensitization. Central sensitization manifests as pain hypersensitivities such as spontaneous pain, allodynia, and hyperalgesia. ${ }^{4}$ Upregulated pGluN1 expression is the evidence of central sensitization, and enhanced pGluN1 expression correlates with the development of painful symptoms. ${ }^{20}$

DEX attenuates nociceptive stimuli and inhibits nociceptive transmission. It inhibits glial cell activation and the release of substance $\mathrm{P}$ and calcitonin gene-related peptide from the spinal cord; the latter initiates central sensitization to pain signals. ${ }^{21}$ The antinociceptive effect of DEX could suppress the development of mechanical allodynia, activation of NMDA receptors, and the ERKs signaling pathway. ${ }^{22,23}$ DEX protects organs from IR injury. Several mechanisms of the protective effect against IR injury of DEX have been explored. First, DEX inhibits catecholamine release in the plasma by activating $\alpha 2$ receptors. ${ }^{6}$ Decreased production of catecholamine prevents overproduction of free radicals. Inhibition of free radical production by DEX may prevent IR injury. ${ }^{24}$ Second, DEX decreases ROS production by blocking xanthine oxidases. ${ }^{25}$ Inhibition of xanthine oxidase scavenges ROS and prevents the development of mechanical allodynia and central sensitization. ${ }^{16}$ Third, DEX may act as a direct and indirect antioxidant. Antioxidants scavenge peroxynitrite, inhibit oxidative stress-induced IR injury, and alleviate developments of mechanical allodynia in CPIP rats. ${ }^{26}$ Fourth, DEX has an anti-inflammatory effect. It decreases inflammatory responses as much as methylprednisolone in spinal cord injury and inflammatory cytokines (TNF- $\alpha$ and IL-6) after IR injury. ${ }^{27}$ Our results suggested that the protective effect against IR injury and antinociceptive effect of DEX could suppress the development of mechanical allodynia, activation of NMDA receptors, and the ERK signaling pathway.

There are some limitations to this study. First, we injected DEX 1 hour before reperfusion. In the skeletal muscle IR injury model, DEX was injected 1 hour before ischemia reduced IR injury. ${ }^{11}$ Thus, we presumed that 1 hour before reperfusion was the ideal injection time; however, further studies are required to evaluate the ideal injection time to prevent allodynia and central sensitization in a rat CPIP model. Second, we did not find the exact mechanisms of DEX to reduce allodynia and central sensitization. Third, we did not examine the contralateral spinal cord. Changes of the ipsilateral spinal cord showed DEX could inhibit central sensitization, but the changes of contralateral spinal cord could give more information to support our results. Fourth, we used small numbers of spinal cord specimens. It would be an apparent weakness of this study. However, spinal cord samples showed significant results despite using limited numbers of samples.

In conclusion, our study demonstrates that DEX administration 1 hour before reperfusion significantly suppresses the development of mechanical allodynia in a CPIP rat model in a dose-dependent manner. This result may be due to the reduction of IR injury-related damage and the antinociceptive effect of DEX. These findings suggest that pretreatment with DEX may be a practical approach for the prevention and attenuation of ischemic and inflammatory pain conditions, including CRPS. 


\section{Author contributions}

SS Park collected, analyzed, and interpreted the data, and critically revised the manuscript. JS Yeo participated in the conception and design of the study, collected, analyzed, and interpreted the data, and wrote and critically revised the manuscript. All authors contributed to data analysis, drafting and revising the article, gave final approval of the version to be published, and agree to be accountable for all aspects of the work.

\section{Disclosure}

The authors report no conflicts of interest in this work.

\section{References}

1. Oerlemans HM, Oostendorp RA, de Boo T, Perez RS, Goris RJ. Signs and symptoms in complex regional pain syndrome type I/reflex sympathetic dystrophy: judgment of the physician versus objective measurement. Clin J Pain. 1999;15(3):224-232.

2. de Mos M, Laferrière A, Millecamps M, et al. Role of NFkappaB in an animal model of complex regional pain syndrome-type I (CRPS-I). J Pain. 2009;10(11):1161-1169.

3. Coderre TJ, Xanthos DN, Francis L, Bennett GJ. Chronic postischemia pain (CPIP): a novel animal model of complex regional pain syndrome-type I (CRPS-I; reflex sympathetic dystrophy) produced by prolonged hindpaw ischemia and reperfusion in the rat. Pain. 2004;112(1-2):94-105.

4. Woolf CJ. Central sensitization: implications for the diagnosis and treatment of pain. Pain. 2011;152(3 Suppl):S2-S15.

5. Zhou HY, Chen SR, Pan HL. Targeting N-methyl-D-aspartate receptors for treatment of neuropathic pain. Expert Rev Clin Pharmacol. 2011;4(3):379-388.

6. Buerkle H, Yaksh TL. Pharmacological evidence for different alpha 2-adrenergic receptor sites mediating analgesia and sedation in the rat. Br J Anaesth. 1998;81(2):208-215.

7. Park HJ, Kim YH, Koh HJ, et al. Analgesic effects of dexmedetomidine in vincristine-evoked painful neuropathic rats. J Korean Med Sci. 2012;27(11):1411-1417.

8. Honda Y, Higuchi H, Matsuoka Y, et al. The inhibitory effect of locally injected dexmedetomidine on carrageenan-induced nociception in rats. Eur J Pharmacol. 2015;764:215-219.

9. Yoshitomi O, Cho S, Hara T, et al. Direct protective effects of dexmedetomidine against myocardial ischemia-reperfusion injury in anesthetized pigs. Shock. 2012;38(1):92-97.

10. Engelhard K, Werner C, Eberspächer E, et al. The effect of the alpha 2-agonist dexmedetomidine and the N-methyl-D-aspartate antagonist $\mathrm{S}(+)$-ketamine on the expression of apoptosis-regulating proteins after incomplete cerebral ischemia and reperfusion in rats. Anesth Analg. 2003;96(2):524-531.
11. Gu J, Sun P, Zhao H, et al. Dexmedetomidine provides renoprotection against ischemia-reperfusion injury in mice. Crit Care. 2011;15(3): R153.

12. Dong X, Xing Q, Li Y, Han X, Sun L. Dexmedetomidine protects against ischemia-reperfusion injury in rat skeletal muscle. J Surg Res. 2014;186(1):240-245.

13. Chaplan SR, Bach FW, Pogrel JW, Chung JM, Yaksh TL. Quantitative assessment of tactile allodynia in the rat paw. $J$ Neurosci Methods. 1994;53(1):55-63.

14. Kwak K-H, Jung KY, Choi JY, et al. Contralateral allodynia and central change in the chronic post-ischemic pain model rats. Korean J Anesthesiol. 2009;56(4):419-424.

15. de Groot H, Rauen U. Ischemia-reperfusion injury: processes in pathogenetic networks: a review. Transplant Proc. 2007;39(2):481-484.

16. Teng D, Hornberger TA. Optimal temperature for hypothermia intervention in mouse model of skeletal muscle ischemia reperfusion injury. Cell Mol Bioeng. 2011;4(4):717-723.

17. Kwak KH, Han $\mathrm{CG}$, Lee $\mathrm{SH}$, et al. Reactive oxygen species in rats with chronic post-ischemia pain. Acta Anaesthesiol Scand. 2009;53(5): 648-656.

18. Jänig W, Baron R. Complex regional pain syndrome: mystery explained? Lancet Neurol. 2003;2(11):687-697.

19. Ji RR, Befort K, Brenner GJ, Woolf CJ. ERK MAP kinase activation in superficial spinal cord neurons induces prodynorphin and NK-1 upregulation and contributes to persistent inflammatory pain hypersensitivity. J Neurosci. 2002;22(2):4780485

20. Gao X, Kim HK, Chung JM, Chung K. Reactive oxygen species (ROS) are involved in enhancement of NMDA-receptor phosphorylation in animal models of pain. Pain. 2007;131(3):262-271.

21. Takano M, Takano Y, Yaksh TL. Release of calcitonin gene-related peptide (CGRP), substance P (SP), and vasoactive intestinal polypeptide (VIP) from rat spinal cord: modulation by alpha 2 agonists. Peptides. 1993;14(2): 371-378.

22. Fan QQ, Li L, Wang WT, Yang X, Suo ZW, Hu XD. Activation of $\alpha 2$ adrenoceptors inhibited NMDA receptor-mediated nociceptive transmission in spinal dorsal horn of mice with inflammatory pain. Neuropharmacology. 2014;77:185-192.

23. Liu L, Ji F, Liang J, He H, Fu Y, Cao M. Inhibition by dexmedetomidine of the activation of spinal dorsal horn glias and the intracellular ERK signaling pathway induced by nerve injury. Brain Res. 2012;1427:1-9.

24. Kocoglu H, Ozturk H, Ozturk H, Yilmaz F, Gulcu N. Effect of dexmedetomidine on ischemia-reperfusion injury in rat kidney: a histopathologic study. Ren Fail. 2009;31(1):70-74.

25. Cosar M, Eser O, Fidan H, et al. The neuroprotective effect of dexmedetomidine in the hippocampus of rabbits after subarachnoid hemorrhage. Surg Neurol. 2009;71(1):54-59.

26. Kwak KH, Jung H, Park JM, et al. A peroxynitrite decomposition catalyst prevents mechanical allodynia and NMDA receptor activation in the hind-paw ischemia reperfusion injury rats. Exp Ther Med. 2014;7(2):508-512.

27. Can M, Gul S, Bektas S, Hanci V, Acikgoz S. Effects of dexmedetomidine or methylprednisolone on inflammatory responses in spinal cord injury. Acta Anaesthesiol Scand. 2009;53(8):1068-1072.
Journal of Pain Research

\section{Publish your work in this journal}

The Journal of Pain Research is an international, peer reviewed, open access, online journal that welcomes laboratory and clinical findings in the fields of pain research and the prevention and management of pain. Original research, reviews, symposium reports, hypothesis formation and commentaries are all considered for publication.

\section{Dovepress}

The manuscript management system is completely online and includes a very quick and fair peer-review system, which is all easy to use. Visit http://www.dovepress.com/testimonials.php to read real quotes from published authors. 